\title{
The Etiology of Mathematical and Reading (Dis)ability Covariation in a Sample of Dutch Twins
}

\author{
Ezra M. Markowitz, ${ }^{1,2}$ Gonneke Willemsen, ${ }^{2}$ Susan L. Trumbetta, ' Toos C. E. M. van Beijsterveldt, ${ }^{2}$ \\ and Dorret I. Boomsma ${ }^{2}$ \\ ' Department of Psychology, Vassar College, Poughkeepsie, United States of America \\ ${ }^{2}$ Department of Biological Psychology, Vrije Universiteit,Amsterdam, the Netherlands
}

\begin{abstract}
$T^{\text {he }}$ he genetic etiology of mathematical and reading (dis)ability has been studied in a number of distinct samples, but the true nature of the relationship between the two remains unclear. Data from the Netherlands Twin Register was used to determine the etiology of the relationship between mathematical and reading (dis)ability in adolescent twins. Ratings of mathematical and reading problems were obtained from parents of over 1500 twin pairs. Results of bivariate structural equation modeling showed a genetic correlation around .60, which explained over $90 \%$ of the phenotypic correlation between mathematical and reading ability. The genetic model was the same for males and females.
\end{abstract}

The phenotypic relationship between mathematical and reading ability, especially in samples selected for cognitive deficits, has received extensive study. In the past 15 years, several studies have investigated the etiology of this relationship. In addition, the independent etiologies of both mathematical and reading (dis)ability have been intensively studied. Estimates of univariate heritability and the genetic correlation between mathematics and reading vary widely. For example, while Alarcón et al. (2000) found a heritability estimate of around $90 \%$ for mathematical performance, other studies have found much lower heritability estimates for mathematical ability (e.g., $\mathrm{h}^{2}=.17$, Thompson et al., $1991 ; \mathrm{h}^{2}=.21$, Wadsworth et al., 1995; $\mathrm{h}^{2}=.65$, Oliver et al., 2004). Similarly, estimates for the heritability of reading ability range from .18 to .81 (Wadsworth et al., 2001). Estimates of the genetic correlation between mathematical and reading (dis)ability also vary, although fewer studies of this relationship have been conducted. For example, Thompson et al. (1991) estimated the genetic correlation between reading and mathematics performance to be .98 in a sample of normally achieving students. Knopik et al. (1997) estimated the bivariate genetic correlation at .53 in a sample selected for mathematical and reading deficits. Studies have also yielded different estimates for the percentage of phenotypic correlation explained by genetic influence. In a sample selected for reading deficits, Light et al. (1998) found that shared heritable influences explained $65 \%$ of the phenotypic correlation between composite verbal and math measures; in a reading-disabled sample, Gillis et al. (1992) report that shared genetic influences explained $98 \%$ of the correlation ( $55 \%$ in a control sample).

As Oliver et al. (2004) suggest, the wide range of estimates for heritability and genetic correlations is most likely due to differences in the samples analyzed, the measures reported on, and the varying ages of the participants. Additionally, the use of different statistical methodologies (e.g., DeFries-Fulker extremes analysis vs. maximum likelihood estimation) may increase the disparity of the estimates. The size of the samples may also be an important factor: a number of previous studies rely on relatively small sample sizes, which increase the confidence intervals around heritability and correlation estimates. Still, the disparities among estimates are great, raising questions about the true relationship between mathematical and reading ability.

Part of the answer may come from studies that have looked beyond mathematical and reading (dis)ability to other cognitive correlates. As Plomin and Craig (2001) point out, an increasing number of studies have been conducted using genetically informative samples to explore the relationship between various cognitive traits. Investigations examining the relationship between reading and other cognitive traits, in particular Verbal and Full Scale IQ (FSIQ), are especially prevalent. Wainwright et al. (2004) report estimates that range from $21 \%$ to $80 \%$ for the percentage of phenotypic correlation between FSIQ and reading ability explained by shared genetic factors, with most estimates closer to the middle and

Received 22 July, 2005; accepted 24 August, 2005.

Address for correspondence: Dorret Boomsma, Biological Psychology, Vrije Universiteit, Van der Boechorststraat 1, 1081 BT Amsterdam, the Netherlands.E-mail: dorret@psy.vu.nl 
top of the range. Studies of the phenotypic correlation between mathematical ability and FSIQ are less abundant, but not completely absent from the literature. For example, Alarcón et al. (2000) find that shared genetic influences account for nearly $90 \%$ of the correlation between mathematical performance and general cognitive ability. Finally, Light et al. (1998) found evidence that the phenotypic and genetic correlations between mathematical and reading deficits can be partially explained by higher order traits, especially in a readingdisabled sample. These studies indicate the need for a careful interpretation of any observed correlation between mathematical and reading (dis)ability.

The importance of understanding the etiology of various cognitive traits and their intercorrelations is far from an academic exercise in statistical methodology. As others have explained (cf. Light et al., 1998), a complete understanding of the influences on mathematical ability, reading, IQ and other cognitive processes will allow for the development of better interventions for individuals who exhibit deficits; furthermore, as the relationships between these traits become clearer, it will be possible to apply more efficient interventions, aimed not only at specific deficits, but also at underlying problems. As Light et al. (1998) suggest, improving an individual's overall verbal and phonologic abilities may ultimately help reduce not only his or her reading problems, but mathematical deficits as well.

In addition to studying the etiology of mathematical and reading ability covariation, this study also explores the relationship between mothers' and fathers' ratings of the same twins. When multiple rater scores are available for analysis, a correlation between ratings can be computed. The size of this correlation provides important information about the validity of the measures, whether raters are measuring the same trait, and whether a rater-bias model should be fitted to the data. A high level of agreement indicates that raters are most likely measuring the same trait. However, when agreement is high, it can be assumed that no informative data is obtained by retaining both raters. When interrater agreement is low, two underlying processes may be occurring (which are not necessarily mutually exclusive). First, raters may be measuring different traits, or at least different aspects of the same underlying trait. This may be due to the fact that raters are exposed to the phenotype(s) in very different situations. Second, the low agreement may be due to rater-specific bias that is nonsituational (e.g., fathers consistently rate children to be more intelligent than do mothers). When interrater agreement is low, the researcher must separate effects of rater-bias from the effects of more 'interesting' factors; this can be accomplished by including multiple rater scores in analyses, using a rater bias model (e.g., Bartels et al., 2004).

Lastly, we are also interested in the possible relationship between the type of school an individual attends and the presence of mathematical and reading problems. We expect that children who attend special education schools will be rated as having more problems in mathematics and reading, with a higher proportion of the children who attend special education rated as having more problems than children who attend 'normal' education. It is also of interest to know whether twins concordant for no cognitive difficulties are also concordant for attending normal schools (and vice versa), as would be expected.

Thus, the present study has three primary objectives. The first is to explore the relationship between the type of school an individual attended and the presence of parent-rated mathematical and reading problems. The second is to assess the relative contributions of genetic and environmental influences to two correlated traits, reading and mathematical (dis)ability. Finally, we are interested in assessing the etiology of the correlation between the traits in terms of shared genetic and environmental influences in a sample of Dutch twins that has not been selected for mathematical or reading deficits. Previous research has produced a wide range of heritability estimates for both traits, as well as for the genetic correlation between them; it is hoped that the use of a large, novel sample that includes opposite-sex twin pairs (which provides a means for directly testing sex-differences in the etiology of each trait) will help clarify the relationship between mathematical and reading (dis)ability.

\section{$\overline{\text { Methods }}$ \\ Participants}

The etiologies of reading and mathematical (dis)ability were explored using data collected in the 1991 wave of the Netherlands Twin-Family Study of Health-Related Behavior. The twin families come from the larger Netherlands Twin Register. Questionnaires were sent and returned by mail (Boomsma et al., 2002). The mean age of the twins in 1991 was 17.7 years $( \pm 2.3$, range $=12-24)$. The questionnaire asked parents to report on the health, lifestyle and personality of their twins. Information regarding the type of education each twin received was collected from the mothers. Mothers' ratings were available for 1577 twin pairs; fathers' ratings were available for 1381 pairs. For a more thorough description of the sample, see Boomsma et al. (1994) and Koopmans et al. (1995).

Zygosity of same-sex twin pairs was based on DNA or blood group polymorphisms for 356 twin pairs in the mothers' data (324 in the fathers'). All other same-sex zygosity determinations were made by questionnaire. Agreement between zygosity assessment using the questions and by DNA markers/blood typing is around $97 \%$ (Willemsen et al., 2005).

\section{Measures}

\section{Type of Schooling}

We were interested in the relationship between the type of school an individual attended and the presence of problems in mathematics and reading. The questionnaire asked mothers to indicate the type of primary and 
secondary school attended by each twin. Mothers were given a number of possible choices, including 'normal school,' 'special education school for children with learning problems,' 'special education school for children with behavior problems,' and 'school for the blind,' among others. In this study, individuals were placed into one of two categories: 'normal education' or 'special education due to learning problems or physical handicap'.

\section{Mathematics and Reading}

Two measures from the 1991 wave of the NTR were selected, 'child has difficulty in mathematics' and 'child has difficulty with reading'. Parents used a 4-point scale to rate each child, where 0 indicated that the child never had problems, and 3 indicated that the child always had problems $(1=$ sometimes, $2=$ often $)$. We reduced the number of categories from 4 to 3 (never, sometimes, often). Preliminary analyses suggested that this was appropriate, as parents used the category always infrequently (prevalence less than $3.5 \%$ ).

\section{Analyses}

Intertwin Concordance Between School Type and Cognitive Problems

To explore twin pair concordance for schooling and cognitive problems, we created two variables from the mothers' data. The first, school concordance, assigned each twin pair to one of three categories: 'concordant normal education', in which both twins attended normal schools; 'discordant education', in which one twin attended normal schools and the co-twin attended special education; and 'concordant special education', in which both twins attended special education. The second variable, math-reading problem concordance, also assigned each twin pair to one of three categories: 'concordant no problems', in which neither twin was rated as having serious problems in mathematics or reading; 'discordant for mathematical and/or reading problems', in which one twin was rated as having serious problems in at least one trait; and 'concordant for mathematical and/or reading problems', in which each twin was rated as having serious problems in at least one trait (or both). 'Serious problem' was defined as a rating of often with respect to 'child has reading difficulty' or 'child has difficulty with mathematics'.

\section{Interrater Agreement}

Polyserial correlations between mothers' and fathers' ratings were computed using Prelis 2.54 (Jöreskog \& Sörbom, 1996) to determine whether mothers and fathers were rating the same aspects of mathematical and reading ability in their twins.

\section{Threshold Models and Polychoric Correlations}

Threshold models and polychoric correlations between twins were computed using maximum likelihood estimation in Mx (Neale et al., 2003). Threshold models are used when the observed data are in an ordinal form but the trait being studied (e.g., mathematical ability) is assumed to have an associated underlying continuous liability. For example, a trait that has two categories (e.g., affected and normal) has one threshold; as measured by the trait, an individual is either affected or normal. A number of factors (genetic or environmental) influence whether or not an individual is affected. If the individual has just enough of each influence, he or she is pushed beyond the threshold, and thus becomes 'affected'. Thus, the underlying liability is a continuous variable. In the present study, both mathematical and reading ability are analyzed using two thresholds, which are computed based on the prevalences of each trait. The variance of the continuous liability was fixed to unity (Falconer, 1981).

\section{Genetic Analyses}

We applied structural equation modeling using the assumptions of the classical twin study in order to decompose the variance of the liability of the two phenotypes into variance due to additive genetics (A), common environment $(\mathrm{C})$ and nonshared environment (E; Boomsma et al., 2002). In the bi- or multivariate case, it is possible to determine the etiology of the correlation between traits. This phenotypic correlation can be decomposed into a part due to shared genetic factors, a part due to shared common environmental factors, and a part due to shared (between traits) unique environmental factors (Duffy \& Martin, 1994; Heath et al., 1993). Using data from male and female twins, it is possible to test whether or not there are sex differences in the etiologies of the traits being studied; this is accomplished by constraining the estimates for males and females to be equal, and then comparing the fit of the constrained model to the fit of the unconstrained model. In addition, including data from opposite-sex twin pairs (DOS) allows us to determine, to some extent, whether or not the same genes are active in males and in females. This can be accomplished by setting the genetic correlation between DOS twins to .5 , as is done for dizygotic (DZ) twins, and comparing the fit of the model to a model in which the correlation is allowed to fluctuate; if the constrained model does not exhibit a significant loss of fit, it can be assumed that the same genes are operating in males and females.

Univariate models are fitted to each trait using $\mathrm{Mx}$ (Neale et al., 2003) and raw data input. The full model in each case included free parameters to be estimated for $\mathrm{A}$ and $\mathrm{C}$ for males and for females. In addition, an estimate of the genetic correlation between oppositesex twins was computed in the full model (this is set to .5 for DZ twins and 1.0 for monozygotic [MZ] twins). Because threshold models were used in which the variance of the liability distribution was fixed at unity, the unique environment variance was obtained using the formula: $e^{2}=1-a^{2}-c^{2}$. Nested models were then run, in which various parameters were set equal to each other (e.g., factor loadings in males are set equal to those in females). The best fitting model was obtained in each case by comparing negative two times the 
Table 1

Cross Tabulation of 'Education Concordance' and 'Cognitive Problems Concordance'

\begin{tabular}{|c|c|c|c|c|}
\hline & Concordant no problems & Discordant, one twin has $\geq 1$ problem & Concordant, each twin has $\geq 1$ problem & Total \\
\hline \multirow[t]{2}{*}{ Concordant normal education } & $70.8 \%$ & $13.4 \%$ & $6.8 \%$ & $90.9 \%$ \\
\hline & $(657)$ & $(124)$ & $(63)$ & (844) \\
\hline \multirow[t]{2}{*}{ Discordant education } & $1.9 \%$ & $2.2 \%$ & $1.1 \%$ & $5.2 \%$ \\
\hline & (18) & $(20)$ & $(10)$ & (48) \\
\hline \multirow[t]{2}{*}{ Concordant special education } & $3.6 \%$ & $1.0 \%$ & $0.8 \%$ & $3.9 \%$ \\
\hline & (19) & (9) & (8) & $(36)$ \\
\hline \multirow[t]{2}{*}{ Total } & $74.8 \%$ & $16.5 \%$ & $8.7 \%$ & $100 \%$ \\
\hline & (694) & $(153)$ & (81) & (928) \\
\hline
\end{tabular}

Note: Columns represent concordant normal, where neither individual has reading or mathematical problems; concordant affected, in which each twin has at least one problem (or two); discordant, in which one twin has at least one problem and the co-twin is completely unaffected. Prevalences $(\%)$ and total number of subjects $(M)$ shown.

log-likelihood (-2LL) of each submodel to that of either the full model or else to the submodel in which it was nested (e.g., AE and CE are compared to ACE). The subsequent test statistic has a $\chi^{2}$ distribution with degrees of freedom equal to the difference of the degrees of freedom between the two models.

Subsequently, a standard bivariate Cholesky decomposition of variance was conducted to determine whether there was any shared genetic component between mathematics and reading (Loehlin, 1996). The Cholesky decomposition provides an estimate of the genetic and environmental correlations between two variables based on the path estimates produced by the decomposition. These are interesting statistics in their own right; however, using the genetic correlation, it is also possible to determine what percentage of the phenotypic correlation is due to shared genetic influences. Confidence intervals $(95 \%)$ were obtained for all estimates.

\section{$\overline{\text { Results }}$}

Intertwin Concordance Between School Type and Cognitive Problems

Table 1 shows a simple cross tabulation of the variables 'school concordance' and 'math-reading problem concordance'. As can be seen, the greatest number of twin pairs fall into the first cell, a reflection of the fact that most participants attended normal schools and were rated by their mothers as having few cognitive problems. The table also shows the low number of participants who attended special education schools. The third row in the first column reflects, almost entirely, twin pairs who attended special education due to medical problems (i.e., blindness, deafness). The high percentage of twin pairs concordant for having no problems $(74.8 \%)$ indicates that the current sample has not been selected for mathematical or reading disability.

\section{Interrater Agreement}

Averaging across all zygosity groups and both twins, paternal and maternal ratings of both mathematics and reading produced a polychoric correlation of .82, suggesting a high level of agreement between parents. Consequently, we do not consider the use of rater-bias models. As expected, the interrater correlations (within-trait) for first-born and second-born twins did not differ significantly.

\section{Prevalences, Thresholds and Polychoric Twin Correlations}

Table 2 shows polychoric twin correlations and exact sample sizes; Table 3 shows prevalences of mathematics and reading problems (from which thresholds were computed). As can be easily seen, MZ correlations were quite high (above .90 in all cases) and DZ and DOS correlations were for the most part around half those of MZs, suggesting a possible AE factor structure. Thresholds for math ability showed that males and females were rated to be quite similar. In contrast, males were rated as having more problems in reading than females, as reflected by significantly lower threshold estimates for males (by both parents).

In all subsequent model fitting that included reading ability, prevalences for males and females were not equated. Overall, the observed prevalences suggest that most individuals were rated as having few cognitive problems. In addition, mothers' and fathers' thresholds appeared quite similar, though mothers were slightly more likely to rate their children more positively.

\section{Genetic Analyses, Univariate}

We fitted an ACE model (with sex differences) to paternal and maternal ratings of mathematical and

\section{Table 2}

Exact Sample Size (Twin Pairs) and Polychoric Twin Correlations for Mathematical and Reading Ability as Rated by Parents

\begin{tabular}{|c|c|c|c|c|c|c|}
\hline \multirow[t]{2}{*}{ Zygosity } & \multicolumn{3}{|c|}{ Fathers } & \multicolumn{3}{|c|}{ Mothers } \\
\hline & $N$ & Math & Read & $N$ & Math & Read \\
\hline MZM & 228 & .94 & .91 & 265 & .90 & .94 \\
\hline DZM & 213 & .41 & .41 & 237 & .48 & .45 \\
\hline MZF & 315 & .96 & .94 & 364 & .93 & .93 \\
\hline DZF & 232 & .41 & .46 & 268 & .25 & .43 \\
\hline DOS-MF & 196 & .43 & .46 & 220 & .42 & .40 \\
\hline DOS-FM & 197 & .45 & .46 & 223 & .38 & .31 \\
\hline
\end{tabular}


Table 3

Prevalences (\%) and Total Number of Subjects $(M$ ) of Mathematical and Reading Problems for All Individuals

\begin{tabular}{lllcrc}
\hline Response & & & \multicolumn{2}{c}{ Reading } \\
& & Males & Females & Males & Females \\
\hline Never: & fathers & $56.4 \%(704)$ & $53.4 \%(780)$ & $54.9 \%(687)$ & $70.5 \%(1033)$ \\
& mothers & $58.6 \%(826)$ & $56.7 \%(951)$ & $58.7 \%(827)$ & $72.6 \%(1218)$ \\
Sometimes: & fathers & $31.8 \%(397)$ & $32.1 \%(469)$ & $30.9 \%(387)$ & $22.2 \%(325)$ \\
& mothers & $30.5 \%(430)$ & $30.2 \%(506)$ & $27.5 \%(388)$ & $20.7 \%(347)$ \\
Often: & fathers & $11.8 \%(148)$ & $14.5 \%(211)$ & $14.2 \%(178)$ & $7.4 \%(108)$ \\
& mothers & $10.9 \%(153)$ & $13.2 \%(221)$ & $13.8 \%(194)$ & $6.7 \%(113)$ \\
\hline
\end{tabular}

reading ability. Subsequently, a number of submodels were fit by imposing various constraints on the data (e.g., $a$ for males equals $a$ for females). In all four cases, the best fitting model was an AE model (respectively, fathers-math, fathers-reading, mothers-math, mothers-reading: $\chi^{2}=4.33, .13,5.04,4.45 ; d f=7,5$, $5,5 ; p=.74,1.00, .66, .49)$, in which all variance was most parsimoniously explained by genetic and nonshared environmental factors. Shared environment (C) could be dropped, suggesting that common environment does not influence either of these traits significantly. Estimates for males and females could be constrained equal to each other and the correlation between genetic factors in opposite-sex twins could be set to .5 without significant loss of fit, indicating that similar etiologies for males and females exist and that the same genes are active in both sexes. Both traits were found to be highly heritable (same order as presented above: $\left.a^{2}=.95, .92, .91, .93\right)$. Confidence intervals $(95 \%)$ indicated overlap between heritability estimates based on mothers' and fathers' data.

\section{Genetic Analyses, Bivariate}

Polychoric correlations between mathematical and reading ability were .62 (fathers' data) and .57 (mothers' data). Cross-twin cross-trait correlations were computed for each zygosity group. There is no reason to believe that the correlation between mathematics-firstborn twin and reading-second born twin should be different from the correlation between reading-firstborn twin and mathematics-second born twin; thus, these correlations were constrained to equal one another. If $\mathrm{MZ}$ twins show higher crosstrait cross-twin correlations than DZ twins, evidence exists for a genetic correlation between traits. Table 4 shows these correlations.

\section{Table 4}

Cross-Twin Cross-Trait Correlations

\begin{tabular}{lcccccc}
\hline & MZM & DZM & MZF & DZF & DOS-MF & DOS-FM \\
\hline Father & .48 & .27 & .69 & .27 & .34 & .39 \\
Mother & .43 & .26 & .51 & .20 & .32 & .18 \\
\hline
\end{tabular}

Using Mx (Neale et al., 2003), a bivariate Cholesky decomposition was performed on each data set to explore the etiology of these correlations. Table 5 shows the fit statistics of the various bivariate models. Again, an AE model fitted the data best for both mothers' and fathers' ratings. Table 6 shows results of bivariate analyses, including genetic and unique environment correlations (with $95 \%$ confidence intervals), as well as heritability estimates for each trait.

Univariate heritability estimates produced by the Choleksy decomposition matched those produced in univariate analyses exactly. Figure 1 shows the path coefficients for $a$ and $e$, a visual representation of the final model that was fit to both data sets, and the computed genetic and environmental correlations (at the bottom). Confidence intervals computed for both correlations show overlap between mothers and fathers. For both data sets, a midsized correlation between genetic factors was observed (fathers $=.64$; mothers $=.58$ ).

Perhaps more interestingly, $96 \%$ of the phenotypic correlation in the fathers' data $194 \%$ in the mothers' data) was due to shared genetic influences, $\operatorname{sqrt}\left(a^{2}{ }_{\text {math }}\right) * r_{\text {genetic }} * \operatorname{sqrt}\left(a^{2}\right.$ reading $) / r_{\text {traits. }}$. To test whether or not the shared genetic and environmental influences are significant, the paths between mathematics and reading were set to zero for $a$ and $e$. This caused a significant worsening of the fit of the model, indicating that the correlations are significant. We also tested the significance of the specific genetic and environmental contributions on the second trait in the Cholesky decomposition by setting the path from reading-specific $\mathrm{A}$ and $\mathrm{E}$ to reading to zero. Doing so also significantly worsened the fit of the model.

\section{$\overline{\text { Discussion }}$}

To explore the relationship between the type of school twins attended and the presence of cognitive problems, two fairly straightforward analyses were performed on the data. First, twin pair concordance for schooling was compared with twin pair concordance for the presence or absence of problems. In order to obtain a general overview of the data, no discrimination was made between the various types of 
Table 5

Fit Statistics for Full Cholesky Model and Submodels Tested on Fathers' Data (Mothers' in Italics)

\begin{tabular}{|c|c|c|c|c|c|c|c|}
\hline Model & -211 & $d f$ & AIC & $\Delta \chi^{2}$ & $\Delta d f$ & $p$ & $\triangle \mathrm{AIC}$ \\
\hline \multirow[t]{2}{*}{ Full, $G=.5$, sex diff. } & 8177.2 & 5405 & -2632.8 & - & - & - & - \\
\hline & 9165.2 & 6152 & -3138.8 & - & - & - & - \\
\hline \multirow[t]{2}{*}{ ACE no sex diff. } & 8183.4 & 5414 & -2644.6 & 6.23 & 9 & .72 & -11.77 \\
\hline & 9169.4 & 6161 & -3152.6 & 4.2 & 9 & .9 & -13.83 \\
\hline \multirow[t]{2}{*}{$\mathbf{A E}$} & 8183.4 & 5417 & -2650.6 & 6.23 & 12 & .90 & -17.77 \\
\hline & 9169.4 & 6164 & -3158.6 & 4.2 & 12 & .98 & -19.83 \\
\hline \multirow[t]{2}{*}{$A E$, no common $A$} & 8598.3 & 5418 & -2237.7 & 421.1 & 13 & $<.001$ & 395.1 \\
\hline & 9514.7 & 6165 & -2815.3 & 349.3 & 13 & $<.001$ & 323.3 \\
\hline \multirow[t]{2}{*}{$A E$, no specific $A$} & 8478.1 & 5418 & -2357.9 & 300.9 & 13 & $<.001$ & 274.9 \\
\hline & 9229.1 & 6165 & -3100.9 & 63.9 & 13 & $<.001$ & 37.9 \\
\hline \multirow[t]{2}{*}{$A E$, no common $E$} & 8207.8 & 5418 & -2628.2 & 30.6 & 13 & .004 & 4.6 \\
\hline & 9600.3 & 6165 & -2729.7 & 435.1 & 13 & $<.001$ & 409.1 \\
\hline \multirow[t]{2}{*}{ CE } & 8534.6 & 5417 & -2299.4 & 336.6 & 12 & $<.001$ & 312.6 \\
\hline & 9552.5 & 6164 & -2775.5 & 387.3 & 12 & $<.001$ & 363.3 \\
\hline \multirow[t]{2}{*}{$E$} & 9207.2 & 5420 & -1632.8 & 1009.3 & 15 & $<.001$ & 979.3 \\
\hline & $10,289.7$ & 6167 & -2044.3 & 1124.5 & 15 & $<.001$ & 1094.5 \\
\hline
\end{tabular}

Note: All models compared to full model. Best fitting model is in bold.

cognitive problems an individual could possibly exhibit; that is, individuals with serious mathematical, reading, or mathematical and reading problems were all simply indicating as having 'cognitive problems.' As predicted, most twin pairs were concordant for both attending 'normal' schools and for having no cognitive problems. In addition, twin pairs in which at least one twin attended special education exhibited higher percentages of cognitive problems. We also expected that a higher proportion of children (individuals) who attended special education would be rated as having serious mathematical and reading problems as compared to children who attended normal schools. Preliminary analyses (not shown) showed a pattern of prevalences that confirmed this expectation. While over $60 \%$ of individuals who attended normal schools were rated as having no mathematical or reading problems, only around $30 \%$ of those attending special education schools were rated problem-free. Conversely, mothers rated fewer than $10 \%$ of individuals who attended normal schools as often having problems in mathematics or reading, while rating more than $30 \%$ of special-education individuals as often having problems.

Although the etiology of the phenotypic relationship between mathematical and reading ability has been studied previously, earlier studies failed to come to consensus regarding the true nature of the relationship. The primary purpose of this study was to explore this relationship in a large sample not selected for reading or mathematical deficits. Specifically, we were interested in determining three primary statistics: heritability estimates for mathematical and reading ability; the size of the correlation between genetic influences that act on both mathematical and reading ability; and the percentage of phenotypic covariation that is explained by these shared genetic factors. Heritability estimates were quite high (above .90 for both traits), indicating very strong genetic influences on both traits. Using a standard bivariate Cholesky decomposition, a medium-size correlation between latent

Table 6

Results of Bivariate Cholesky Analysis of Mathematical and Reading Ability

\begin{tabular}{|c|c|c|c|c|c|c|}
\hline \multirow[b]{2}{*}{ Rater } & \multirow[b]{2}{*}{$r(a)$} & \multirow[b]{2}{*}{$r(e)$} & \multicolumn{2}{|c|}{$a^{2}$} & \multicolumn{2}{|c|}{$e^{2}$} \\
\hline & & & Math & Read & Math & Read \\
\hline \multirow[t]{2}{*}{ Fathers } & .64 & .63 & $.95(.94-.96)$ & $.92(.90-.94)$ & $.05(.04-.06)$ & $.08(.06-.10)$ \\
\hline & $(.60-.68)$ & $(.40-.82)$ & & & & \\
\hline \multirow[t]{2}{*}{ Mothers } & .58 & .80 & $.91(.89-.93)$ & $.93(.91-.95)$ & $.09(.08-.11)$ & $.07(.05-.09)$ \\
\hline & $(.54-.62)$ & $(.63-.91)$ & & & & \\
\hline
\end{tabular}

Note: $95 \%$ confidence intervals shown in parentheses. 


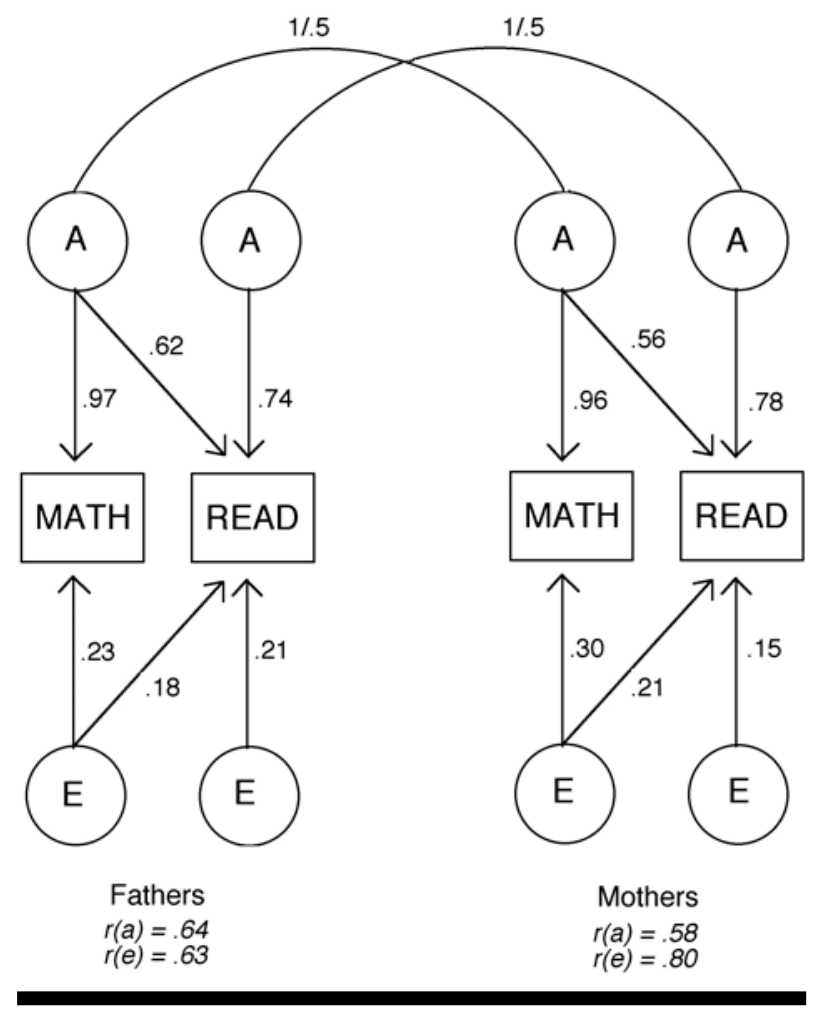

Figure 1

Bivariate Cholesky decomposition of correlation between mathematical and reading ability as rated by mothers and fathers separately. Path estimates on left are from fathers' data; on the right, from mothers'. The diagram itself represents the full model, with Twin 1 on the left, and Twin 2 on the right.

genetic factors (.64 in fathers' ratings, .58 in mothers') was found. The most straightforward interpretation of this correlation is that approximately $60 \%$ of the genetic factors that influence mathematical (dis)ability also influence reading (dis)ability. Furthermore, approximately $95 \%$ of the phenotypic correlation between mathematical and reading ability was explained by these shared genetic influences, with the remaining 5\% explained by nonshared environmental influences. Thus, although there is considerable variation in the genes affecting each trait, the portion that does overlap explains essentially all of the relationship between phenotypes.

The findings of this study lend support to previous findings of high heritabilities for both mathematical and reading ability. Interestingly, most high estimates for these heritabilities have been reported in samples selected for some sort of cognitive disability or deficit (usually reading), while estimates in 'normal' populations have tended to be somewhat lower (though the pattern of previous results is not totally clear). Our results suggest that both mathematical and reading ability are highly heritable even in a sample not selected for disability. Consistent with some but not all previous studies, no influences of shared environment were found, suggesting that variation between families in learning environments has limited influence on variation in mathematical and reading skills.

The use of parental ratings of mathematical and reading disability provided both advantages and disadvantages. The fairly high level of interrater agreement (above $80 \%$ ) suggests that parents agreed much of the time on the severity of mathematical and reading problems that their twins have, and secondly, that the measures selected are most likely valid representations of underlying mathematical and reading (dis)ability in this sample of Dutch twins. Because parents observe their children's performance over a long period of time and in great depth, parental ratings have somewhat of an advantage over one-time objective measures of achievement, which are highly influenced by situational factors; additionally, parental ratings may in fact reflect, at least in part, parents' knowledge of objective information about the twins (e.g., test scores). Research by Dewey et al. (2003) also supports the validity of parental ratings of cognitive ability in children. In addition, one of the primary advantages of using parental ratings here was the large sample size available for data analysis in this study.

Additionally, a related and secondary finding has some potentially interesting and informative implications. We observed an interesting pattern in the prevalences for mathematics and reading: parents rated daughters as having substantially fewer problems in reading than sons. This finding is consistent with previous research indicating that females are better readers. However, an alternative explanation exists as well: perhaps parents rate daughters better in reading due to the widely held stereotype that women are better readers than men. At the same time, mothers and fathers did not rate boys as better in mathematics than girls, although if the parents were simply following societal stereotypes, we might have expected to see such an effect. Taken together, these findings seem to imply both the possible disappearance of the 'boys are better in math' stereotype in the Netherlands and that girls are better readers, although we cannot say anything definitive about the observed differences.

Lastly, the implications of the measures used in these analyses warrant further consideration. Although the validity of the measures is strongly supported by the high level of interrater agreement, the results of the cross tabulation of schooling and cognitive problems, and the observed distribution of cognitive problems as a function of the individuals' schooling, a valid question remains: what was truly measured and analyzed in this study? The most accurate description of the 'surface' phenotype studied may be: 'the heritability of being rated by your parents as having mathematical and reading problems'. Thus, in a sense what was studied was the heritability of a child making a specific sort of 'impression' on his or her parents with regards to mathematical and reading ability. This is by no means a less interesting trait than a 'pure' measure of mathematical and reading (dis)ability. 
In many ways, our findings are a strong affirmation of previous results because they were not obtained simply by applying previous methodologies to a new sample; instead, high heritability estimates and a fairly strong genetic correlation were found using a novel sample and measures that are perhaps less than ideal. If anything, this points to the robustness of the findings presented here, and provides strong evidence that the genetic influences on both mathematics and reading are quite significant. Still, it was a concern that perhaps the high heritability estimates were artifacts of the data set itself. To test this, a behavioral trait (short-temperedness) also rated by parents was selected for analysis from the same questionnaire. Results showed a considerably lower heritability estimate as well as a shared environmental component, which helped to allay concerns regarding the validity of the questionnaire.

Current and future research in the etiology of complex behavioral and cognitive traits appears to be moving toward the identification of specific genes that may be responsible for the variation in these traits. However, continued study of heritability and genetic correlations between various cognitive traits is not simply old news. The more accurate the picture is of the relationships between these various traits, the more confident we can be in directing future research in the correct direction.

\section{$\overline{\text { Acknowledgments }}$}

The authors acknowledge with appreciation Vassar College's Undergraduate Research Summer Institute and Dr. Peter Pappas, its Director, for their support of this project. We would also like to thank all of the members of the Biological Psychology department at the Vrije University in Amsterdam who helped with this project. Support from the Netherlands Organization for Scientific Research (NWO 575-25006) is gratefully acknowledged.

\section{$\overline{\text { References }}$}

Alarcón, M., Knopik, V. S., \& DeFries, J. C. (2000). Covariation of mathematics achievement and general cognitive ability in twins. Journal of School Psychology, 28, 63-77.

Bartels, M., Boomsma, D. I., Hudziak, J. J., Rietveld, M. J., van Beijsterveldt, T. C., \& van den Oord, E. J. (2004). Disentangling genetic, environmental, and rater effects on internalizing and externalizing problem behavior in 10-year-old twins. Twin Research, 7, 162-175.

Boomsma, D. I., Vink, J. M., van Beijsterveldt, C. E. M., de Geus, E. J. C., Beem, A. L., Mulder, E. J. C. M., Derks, E. M., Riese, H., Willemsen, G. A., Bartels, M., van den Berg, M., Kupper, N. H., Polderman, T. J., Posthuma, D., Rietveld, M. J., Stubbe, J. H., Knol, L. I., Stroet, T., \& van Baal, G. C. (2002). Netherlands Twin Register: A focus on longitudinal research. Twin Research, 5, 401-406.
Boomsma D. I., Koopmans, J. R., van Doornen, L. J., \& Orlebeke, J. F. (1994). Genetic and social influences on starting to smoke: A study of Dutch adolescent twins and their parents. Addiction, 89, 219-226.

Dewey, D., Crawford, S. G., \& Kaplan, B. J. (2003). Clinical importance of parent ratings of everyday cognitive abilities in children with learning and attention problems. Journal of Learning Disabilities, 36, 87-95.

Duffy, D. L., \& Martin, N. G. (1994). Inferring the direction of causation in cross-sectional twin data: Theoretical and empirical considerations. Genetic Epidemiology, 11, 483-502.

Falconer, D. S. (1981). Introduction to quantitative genetics (2nd ed.). London: Longman.

Gillis, J. J., DeFries, J. C., \& Fulker, D. W. (1992). Confirmatory factor analysis of reading and mathematics performance: A twin study. Acta Geneticae Medicae et Gemellologiae, 41, 287-300.

Heath, A. C., Kessler, R. C., Neale, M. C., Hewitt, J. K., Eaves, L. J., \& Kendler, K. S. (1993). Testing hypotheses about direction of causation using cross-sectional family data. Behavior Genetics, 23, 29-50.

Jöreskog, K., \& Sörbom, D. (1996). Prelis 2: User's reference guide. Chicago: Scientific Software International.

Knopik, V. S., Alarcón, M., \& DeFries, J. C. (1997). Comorbidity of mathematics and reading deficits: Evidence for a genetic etiology. Behavior Genetics, 27, $447-453$.

Koopmans, J. R., Boomsma, D. I., Heath, A. C., \& van Doornen, L. J. (1995). A multivariate genetic analysis of sensation seeking. Behavior Genetics. 25, 349-356.

Light, J. G., DeFries, J. C., \& Olson, R. K. (1998). Multivariate behavioral genetic analysis of achievement and cognitive measures in reading-disabled and control twin pairs. Human Biology, 70, 215-237.

Loehlin, J. C. (1996). The Cholesky approach: A cautionary note. Behavior Genetics, 26, 65-69.

Neale, M. C., Boker, S. M., Xie, G., \& Maes, H. (2003) Mx: Statistical modeling (6th ed.). Richmond, VA: Department of Psychiatry, Medical College of Virginia.

Oliver, B., Harlaar, N., Thomas, M. E. H., Kovas, Y., Walker, S. O., Petrill, S. A., Spinath, F. M., Dale, P. S., \& Plomin, R. (2004). A twin study of teacher-reported mathematics performance and low performance in 7year-olds. Journal of Educational Psychology, 96, 504-517.

Plomin, R., \& Craig, I. (2001). Genetics, environment and cognitive abilities: Review and work in progress towards a genome scan for quantitative trait locus associations using DNA pooling. British Journal of Psychiatry, 178(Suppl. 40), s41-s48.

Thompson, L. A., Detterman, D. K., \& Plomin, R. (1991). Associations between cognitive abilities and scholastic achievement: Genetic overlap but environmental differences. Psychological Science, 2, 158-165. 
Wadsworth, S. J., Corley, R. P., Hewitt, J. K., \& DeFries, J. C. (2001). Stability of genetic and environmental influences on reading performance at 7,12 , and 16 years of age in the Colorado Adoption Project. Behavior Genetics, 31, 353-359.

Wadsworth, S. J., DeFries, J. C., Fulker, D. W., \& Plomin, R. (1995). Covariation among measures of cognitive ability and academic achievement in the Colorado Adoption Project: Sibling analysis. Personality and Individual Differences, 18, 63-73.
Wainwright, M., Wright, M. J., Geffen, G. M., Geffen, L. B., Luciano, M., \& Martin, N. G. (2004). Genetic and environmental source of covariance between reading tests used in neuropsychological assessment and IQ subtests. Behavior Genetics, 34, 365-376.

Willemsen, G., Posthuma, D., \& Boomsma, D. I. (2005). Environmental factors determine where the Dutch live: Results from the Netherlands Twin Register. Twin Research and Human Genetics, 8, 312-317. 